\title{
Caracterização morfológica dos dentes de mocó Kerodon rupestris: Mammalia: Rodentia
}

Juliana Montovani

\section{THOMAZ $^{1}$}

Ana Flávia de CARVALHO ${ }^{1}$

Maria Angélica MIGLINO²

Celina Almeida Furlanetto

MAÇANARES ${ }^{1}$

Carlos Eduardo

AMBRÓSIO ${ }^{1,2}$

Moacir Franco de OLIVEIRA ${ }^{3}$

\section{Correspondência para:}

ANA FLÁVIA DE CARVALHO

Departamento de Ciências Morfológicas

Centro Universitário da Fundação de Ensino

Octávio Bastos

Avenida Doutor Octávio Bastos, s/n 13874-148 - São João da Boa Vista -SP vetlab03@feob.br

Recebido para publicação: 11/02/2004 Aprovado para publicação: 01/06/2005

\author{
1 - Departamento de Ciências Morfológicas do Centro Universitário da \\ Fundação de Ensino Octávio Bastos, São João da Boa Vista - SP \\ 2 - Departamento de Cirurgia da Faculdade de Medicina Veterinária e \\ Zootecnia da Universidade de São Paulo, São Paulo - SP \\ 3 - Departamento de Medicina Veterinária da Escola Superior de Agricultura \\ de Mossoró, Mossoró - RN
}

\section{Resumo}

O Kerodon rupestris, também conhecido como mocó, é um roedor herbívoro encontrado no Brasil. A dentição dos mamíferos apresentase variável de acordo com as espécies embora seja formada pelos mesmos componentes: esmalte, dentina, cemento e polpa. Neste estudo foram utilizadas sete cabeças de Kerodon rupestris, adultos, de ambos os sexos. Na cavidade oral do Kerodon rupestris, devido ao tamanho relativamente grande dos incisivos, estes se destacaram dos demais e foram encontrados um par em cada mandíbula. Dois pares de pré-molares e três pares de molares foram encontrados, sendo representados na fórmula $2 \mathrm{x}$ (I 1/1, C 0/0, P 1/1, M 3/3). Os molares apresentaram duas cúspides, conformação que conferia a estes dentes um aspecto serrilhado. Microscopicamente os dentes incisivos, prémolares e molares foram classificados como hipsodontes, ou seja, dentes em constante erupção.

\section{Introdução}

O mocó (Kerodon rupestris) pertence à Ordem Rodentia, Família Caviidae, Subfamília Caviinae e Gênero Kerodon ${ }^{11}$. É um roedor herbívoro encontrado na região nordeste do Brasil, habitando ambientes rochosos. Seu peso varia entre 900 a $1000 \mathrm{~g}$, sendo o maior exemplar da família². Embora os dentes destes animais sejam formados pelos mesmos componentes, ou seja, esmalte, dentina, cemento e polpa, a dentição de mocó e de outros mamíferos, em geral, apresenta-se variável de acordo com as espécies ${ }^{3}$. Assim sendo, mamíferos domésticos apresentam dentes classificados como heterodontes, pois há diversos grupos diferentes de dentes, cada um sendo possuidor de determinadas características adaptadas a funções específicas ${ }^{4}$.

Os dentes incisivos dos mamíferos que roem são enormemente aumentados e crescem ininterruptamente ao longo de suas vidas $^{5}$. Estes dentes geralmente são amarelos ou alaranjados e muitas vezes podem apresentar um crescimento além do normal, levando a possíveis problemas clínicos ${ }^{6}$. Os dentes caninos são proeminentes nos carnívoros e ausentes nos roedores. Os prémolares e molares são dentes de perfuração ou de corte sendo destinados ao processamento do alimento ${ }^{4}$. Logo, em muitos herbívoros toda a coluna de dentes pós-caninos é utilizada para a mastigação generalizada e os pré-molares foram modificados se parecendo muito com molares, no processo conhecido como molarização ${ }^{5}$.

Os molares de camundongos apresentam pares de cúspides arredondados estando arranjados em linhas transversais. Os ratos silvestres possuem molares com cúspides triangulares alternadas arranjadas em ziguezague ${ }^{7}$. Alguns mamíferos herbívoros apresentam um espaço entre os dentes 
incisivos e os dentes molariformes conhecido como diastema, sendo responsável pelo deslocamento do aparelho cortador para longe da face, permitindo ao focinho penetrar em aberturas estreitas do meio ambiente em que vivem. ${ }^{8}$

Os dentes podem ser classificados em braquidontes ou hipsodontes. Dentes braquidontes possuem coroa baixa e são adaptados a uma dieta mais branda, que produz menos desgaste dental. Já os hipsodontes, encontrados nos ruminantes, roedores e eqüinos, apresentam-se em constante erupção, não possuindo coroa, colo e raiz definitivos. Os roedores apresentam dentes incisiformes hipsodontes $^{3}$. Dentes molares hipsodontes se parecem com os dentes molares normais quando observados na mandíbula, mas a divisão entre coroa e raiz dentais não é visível. As coroas dos dentes hipsodontes penetram nos ossos mandibulares, sendo o crânio altamente modificado para acomodá-las ${ }^{5}$.

Segundo Crossley et al. ${ }^{9}$, os dentes pré-molares e molares de chinchilas (Chichilla laniger) são hipsodontes in natura, continuando o crescimento do início até o final da vida. Estes roedores apresentam a fórmula dentária 2x (I 1/1, C 0/0, P 1/1, $\mathrm{M} 3 / 3)^{10}$. Contudo, em alguns roedores os molares são enraizados e param de crescer quando ficam adultos os animais ${ }^{6}$.

As estruturas mineralizadas do dente são: o esmalte, dentina e cemento. $\mathrm{O}$ esmalte é um tecido epitelia densamente calcificado e acelular sendo produzido pelos ameloblastos, células que morrem antes da erupção dental ${ }^{11}$. Alguns roedores apresentam esmalte somente na superfície rostral do dente, produzindo uma extremidade cortante auto-afiadora e diretamente relacionada com o hábito de roer destes animais ${ }^{5}$. A dentina é uma estrutura conjuntiva semelhante ao osso e sua matriz orgânica é sintetizada por odontoblastos, células que revestem a superfície interna da dentina $^{3}$. O cemento é uma estrutura também conjuntival, porém menos rígida e * Processo $n^{\circ}$ 0204.002405/01-32, licença número 0001/2001. cementoblastos são as células responsáveis por sua produção ${ }^{4}$. A porção central do dente, conhecida como cavidade pulpar, possui formato aproximado ao próprio dente e é preenchida por tecido conjuntivo frouxo, apresentando vasos e nervos ${ }^{1}$.

Em ratos, nenhuma diferença foi encontrada entre os incisivos superiores e inferiores quanto ao conteúdo e a organização básica das células dentais ${ }^{14}$.

Sendo assim, o presente trabalho objetiva analisar morfologicamente os dentes de Kerodon rupestris através de estudos macro e microscópicos com a finalidade de classificá-los.

\section{Resultados e Discussão}

Neste trabalho foram utilizadas sete cabeças de mocós, Kerodon rupestris, adultos e de ambos os sexos, as quais foram cedidas pelo Centro de Multiplicação de Animais Silvestres (CEMAS), criado pela Escola Superior de Agricultura de Mossoró (ESAM), localizada no Estado do Rio Grande do Norte. IBAMA - Natal-RN*. Após a eutanásia dos animais, suas cabeças foram perfundidas e pós-fixadas com solução de paraformaldeído a 4\% em tampão fosfato de sódio $0,1 \mathrm{M}(\mathrm{pH} 7,4)$. Posteriormente à fixação, dois animais tiveram suas cavidades orais exposta com uso de pinça anatômica, tesoura e bisturi, para que fosse realizada a documentação fotográfica, in situ. Duas cabeças retiradas de um macho e uma fêmea, respectivamente, foram macerados utilizando-se a técnica usual de maceração a frio. Após este procedimento, os crânios foram fotografados para evidenciar e caracterizar a posição dos dentes.

Amostras de dentes molar e incisivo, respectivamente, foram coletadas e processadas pela técnica de desgaste abrasivo e observados ao microscópio óptico e outros dois dentes, também um incisivo e um molar, foram submetidos a técnica de descalcificação modificada ${ }^{11}$, sendo o descalcificador usado formado pela mistura de $800 \mathrm{ml}$ de água destilada, $120 \mathrm{ml}$ de ácido 
fórmico, $80 \mathrm{ml}$ de ácido clorídrico, para um litro. Após a descalcificação química os materiais foram processados pela técnica rotineira de inclusão em um produto similar à parafina (Histosec ${ }^{\circledR}$ - Merck), cortado em micrótomo Leica RM2165 ${ }^{\circledR}$. Cortes de 5 ìm de espessura foram corados com $\mathrm{H} / \mathrm{E}^{13}$. De todo material preparado para a microscopia óptica fizeram-se fotomicrografia em um Fotomicroscópio Nikon ${ }^{\mathrm{TM}}$ E-400 (Japão).

\section{Conclusões}

Os dentes pré-molares e os molares de mocó apresentaram-se macroscopicamente semelhantes entre si, processo que, segundo Pough, Janis e Heiber, é denominado molarização, ou seja, os prémolares foram modificados, assemelhandose aos molares, apresentando ambos os dentes a função de macerar alimentos duros e fibrosos (Figura 1). Além disso, na cavidade oral do Kerodon rupestris, entre os dentes incisivos e os pré-molares foi observado um espaço, um diastema alongado, assim como descrito por Pough Heiber e Mc Farland, sendo este espaço maxilar responsável pelo deslocamento do aparelho cortador para longe da face, permitindo ao focinho penetrar em aberturas estreitas, ou então chegar próximo ao solo durante a sua alimentação (Figura 1).

Em cada mandíbula e maxila de Kerodon rupestris foi observado um par de dentes incisivos, um par de pré-molares e três pares de molares, totalizando 20 dentes os quais foram expressos pela fórmula $2 \mathrm{x}$ (I 1/1, C 0/0, P 1/1, M 3/3), sendo semelhante à fórmula dentária de outro roedor (Chichilla laniger) como foi descrita por Crossley ${ }^{10}$. Ademais, de acordo com Clark e Olfert ${ }^{6}$, os dentes incisivos dos roedores são geralmente amarelos ou alaranjados e estão relacionados ao hábito de roer destes animais, o que pôde ser observado nos mocós estudados, onde os incisivos destacaram-se pelo tamanho relativamente grande quando comparado aos seus outros próprios dentes, apresentan-do coloração alaranjada

Os pré-molares e os molares de Kerodon rupestris caracterizaram-se pela presença de duas cúspides, conformação que conferia um aspecto serrilhado, diferente, por exemplo, dos dentes homônimos de camundongos e ratos silvestres os quais possuem, respectivamente, pré-molares arredondados com pares de cúspides arranjadas em linhas transversais e molares com cúspides triangulares alternadas e arranjadas em ziguezague ${ }^{7}$ (Figura 1).

Ao observar radiografias craniofaciais dos animais estudados, a separação em coroa, colo e raiz dentárias não pôde ser observada, o que caracterizou os dentes como hipsodontes, de acordo com Banks ${ }^{3}$ e Pough, Janis e Heiser ${ }^{5}$, onde os dentes hipsodontes apresentam-se em constante erupção, não possuindo coroa, colo e raiz bem definidos e definitivos, sendo caracterizados por possuir apenas raiz. Outrossim, segundo Crossley et al. ${ }^{9}$, os dentes pré-molares e molares de chinchila (Chichilla laniger) são hipsodontes in natura, continuando o crescimento e erupção desde o inicio até o final da vida, o que também acontece com o Kerodon rupestris, já que possui dentes hipsodontes em todas as categorias, ou seja, incisivos, pré-molares e molares.

Os mocós estudados apresentaram dentes molariformes com as camadas, da região externa para a interna: cemento, esmalte, dentina e polpa bem definidas, sendo as três primeiras, as estruturas mineralizadas do dente, assim como descrito por Gartner e Hiatt ${ }^{11}$ (Figura 2). Foi possível observar os cementoblastos, células responsáveis pela produção de cemento e odontoblastos, responsáveis pela produção da pré-dentina, sendo também observados túbulos da dentina (Figura 3). Os dentes incisivos apresentaram esmalte somente na superfície rostral, assim como foi descrito por Pough, Janis e Heiser. Ainda, de acordo com Smith ${ }^{4}$, em ratos, nenhuma diferença foi encontrada entre os incisivos superiores e inferiores com relação ao conteúdo e a organização básica das células, o que também 


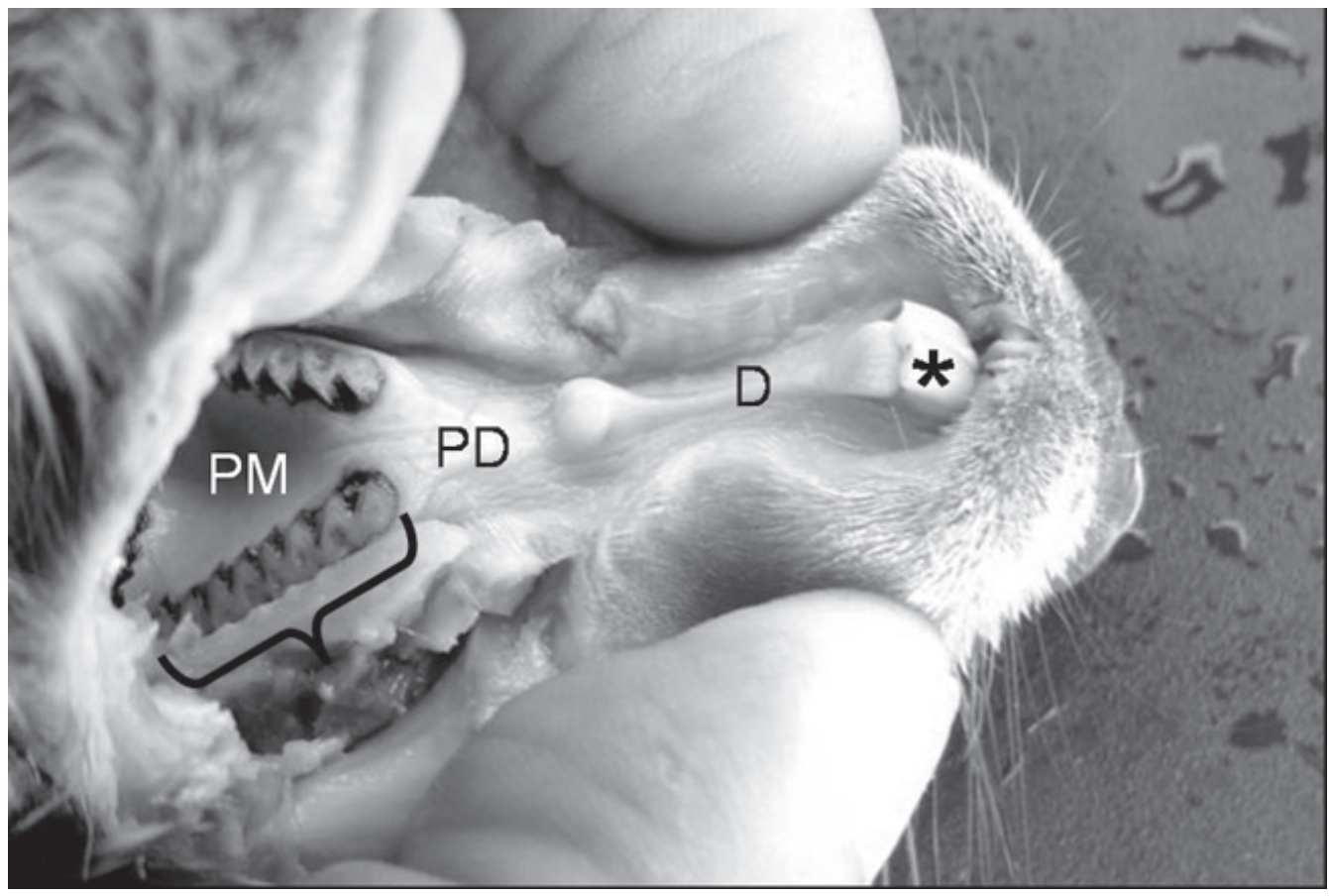

Figura 1 - Região dorsal da cavidade oral de mocó, Kerodon rupestris, onde podem ser observados os dentes incisivos (i), os pré-molares e molares (\}); o lábio maxilar (ls), diastema (d), pálato mole (pm) e pálato duro (pd).

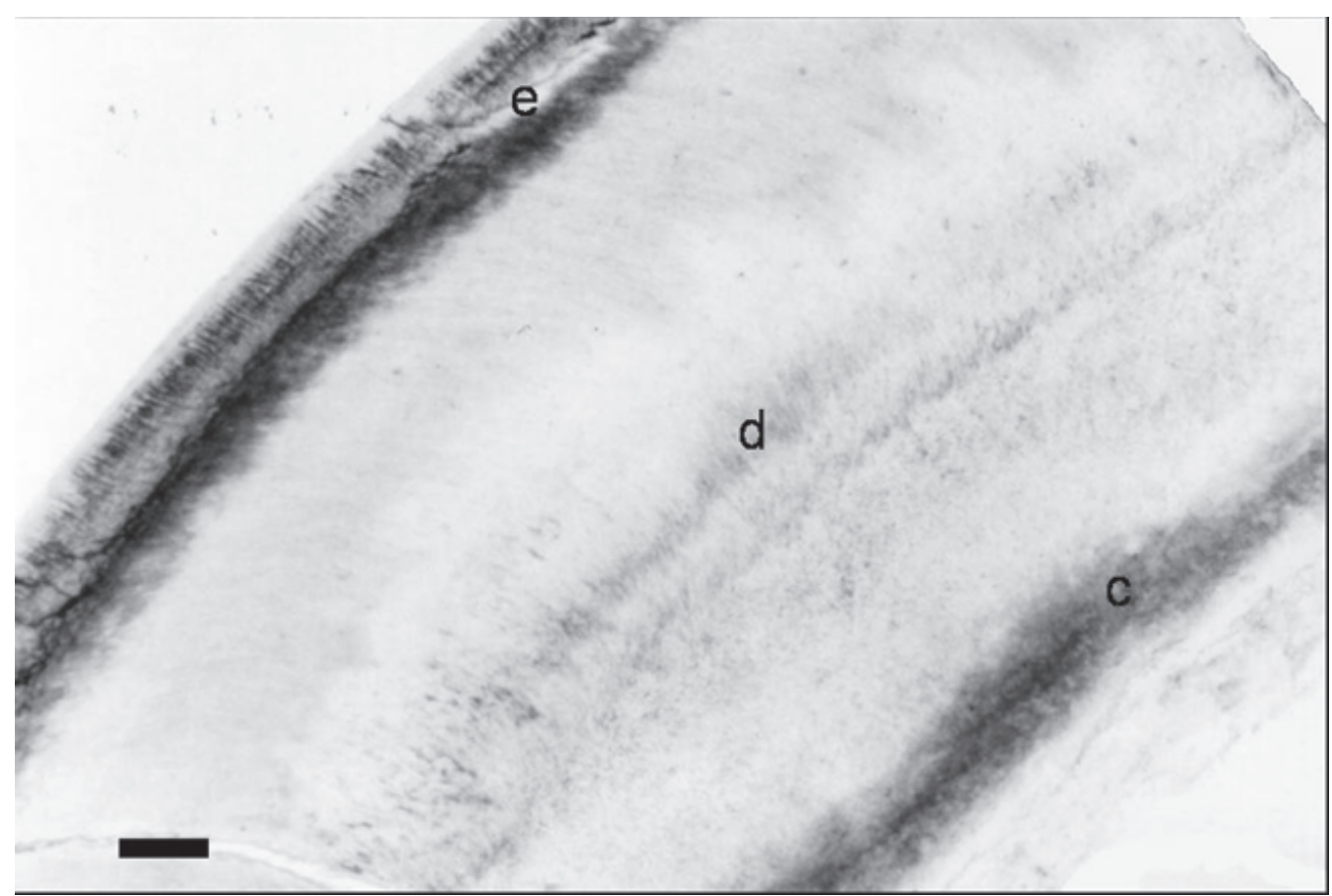

Figura 2 - Dente incisivo mostrando o esmalte (e), dentina (d) e cemento (c). Técnica de desgaste, 44x, barra $=220 \mu \mathrm{m}$. 


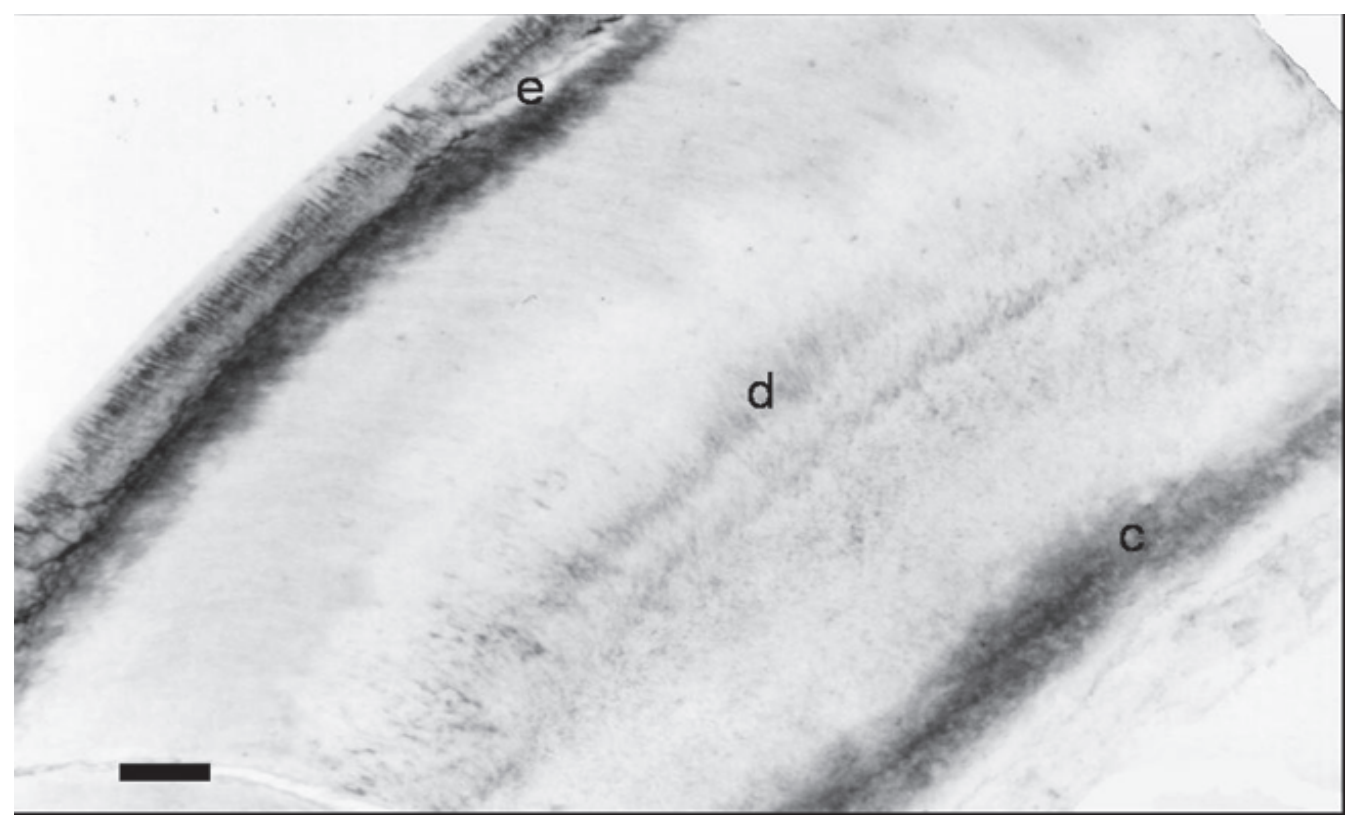

Figura 3 - Área próxima à raiz de um dente molar mostrando cemento (c); dentina (d); polpa (p); ecementoblastos (seta). Técnica de desmineralização, Hematoxilina-eosina, $110 x$, barra $=90 \mu \mathrm{m}$

foi observado em Kerodon rupestris.

Sendo assim, na cavidade oral do mocó, os dentes incisivos destacaram-se devido ao tamanho relativamente grande e foram encontrados um par em cada mandíbula. Dois pares de pré-molares e três pares de molares foram encontrados em cada mandíbula, totalizando 20 dentes. Os molares apresentaram duas cúspides, conformação que conferia a estes dentes um aspecto serrilhado. Microscopicamente os dentes incisivos, pré-molares e molares foram classificados como hipsodontes, ou seja, dentes em constante crescimento. Através da classificação morfológica dos dentes de mocó, foi possível notar a semelhança encontrada com os dentes de chinchilas.

\section{Teeth morphologic characterization of rock cavy Kerodon rupestris: Mammalia: Rodentia}

\section{Abstract}

The Kerodon rupestris, also known as rock cavy, is a herbivorous rodent found in Brazil. The mammalian dentition presented an usual pattern in all species, however each tooth had been formed by the same structural components, e.g. enamel, dentine, cementum and pulp. A molar and incisive teeth samples were collected and processed by the technique of consuming and other teeth had been submitted to the decalcification technique. Thereafter the materials were processed by the histologic routine technique of inclusion in a similar product to paraffin, cut and stained by H/E. All materials prepared for light microscopy were documentated. In the oral cavity of the Kerodon rupestris, the incisors had to be detached of others and had been found a pair in each jaw. Two pairs of premolars and three pairs of molars had been found, being represented in formula $2 x(\mathrm{I} 1 / 1, \mathrm{C} 0$ / $0, \mathrm{P} 1 / 1, \mathrm{M} 3 / 3)$. The molars had two cuspids and this conformation
Key-words:

Kerodon rupestris. Dental materials.

Teeth morphology.

Types of teeth. 
conferred to these teeth a serrated aspect. Microscopically, incisive, premolars and molars teeth had been classified as hypsodonts, teeth in constant eruption.

\section{Referências}

3 BANKS, W. J. Histologia veterinária aplicada. 2 ed. São Paulo: Manole, 1992.

13 BEHMER, O. A.; TOLOSA, E. M. C.; NETO, A. G. F. Manual de técnicas para histologia normal e patológica. São Paulo: Edart, 1976.

6 CLARCK, J. D.; OLFERT, E. D. Rodents (Rodentia). In: FOWLER, M. E. Zoo \& wild animal medicine. 2 ed. Canadian: Saunders, 1986. Cap 46, p. 727-747.

10 CROSSLEY, D. A. Dental disease in chinchillas in the UK. Journal of Small Animal Practice, v. 42, p. 1219, 2001.

9 CROSSLEY, D. A.; et al. Use of computed tomography to investigate cheek tooth abnormalities in chinchillas (Chinchilla laniger). Journal of Small Animal Practice, v. 39, p. 385-389, 1998.

11 GARTNER, L. P.; HIATT, J. L. Tratado de histologia. Rio de Janeiro: Guanabara Koogan, 1997.

2 NETO, E. J. Morphology of the regions ethmoidalis and orbitotemporalis in Galea musteloides (Meyen 1832) and Kerodon rupestris (Wied-Neuwied 1820) (Rodentia: Caviidae) with comments on the phyligenetic systematics of the Caviidae. Journal of Zoological Systematics and Evolutionary Research, v. 38, p. 219229, 2000.

7 POLLY, P. D. Development and evolution occlude: Evolution of development in mammalian teeth. Proceedings of the National Academy of Sciences of the United States of America, v. 97, n. 26, p. 14019$14021,2000$.

8 POUGH, F. H.; HEISER, J. B.; MCFARLAND, W. N. A vida dos vertebrados. 2 ed. São Paulo: Atheneu, 1999.

5 POUGH, F. H.; JANIS, C. M.; HEISER, J. B. A vida dos vertebrados. 3 ed. São Paulo: Atheneu, 2003.

12 RODRIGUES, H. Técnicas anatômicas. 2 ed.. Vitória: Arte Visual, 1998.

1 ROWE, D. L.; HONEYCUTT, R. L. Phylogenetic relationships, ecological correlates, and molecular evolution within the Cavioidea (Mammalia, Rodentia). Molecular Biology and Evolution, v. 19, n. 3, p. 263$277,2002$.

14 SISSON, S. Aparelho Digestório- Parte Geral. In: GETTY, R. Anatomia dos animais domésticos. 5 ed. Rio de Janeiro: Guanabara Koogan, 1986. v. 1, cap. 7, p. 105-107.

4 SMITH, C. E. Histological and three dimensional organization of the odontogenic organ in the upper incisor of $100 \mathrm{gm}$ rats: comparison with the lower incisor. American Journal of Anatomy, v. 142, n. 4, p. 431-455, 1975. 